\title{
QUALITATIVE PROPERTIES OF SOLUTIONS OF CERTAIN FOURTH ORDER LINEAR DIFFERENTIAL EQUATIONS
}

\author{
W.E. TAYLOR, JR. \\ Department of General Academics, Texas A\& M at Galveston \\ Galveston, Texas 77553 U.S.A.
}

(Received January 23, 1980)

ABSTRACT. This work considers differential equations of the form

$$
\left(p y^{\prime \prime}\right) "+q y^{\prime \prime}+r y=0
$$

where $p, q$ and $r$ are positive continuous functions defined on $[0, \infty)$. The main concentration is on the oscillatory and asymptotic behavior of the solutions. Such an investigation is important because the above equation often arises in the study of mechanical vibrations.

KEY WORDS AND PHRASES. Oscillatory solutions, nonoscillatory solutions, strong oscillation, oscillation numbers.

1980 MATHEMATICS SUBJECT CLASSIFICATION CODE. $34 \mathrm{C} 10$.

\section{INTRODUCTION.}

In this paper we are concerned with the asymptotic and oscillation properties of solutions of the differential equation

$$
\left(p y^{\prime \prime}\right) "+q y^{\prime \prime}+r y=0 \text {. }
$$

The following assumptions will be made:

$\left(\mathrm{H}_{0}\right) \mathrm{p}, \mathrm{q}, \mathrm{r}$ are continuous real-valued functions defined on $[0, \infty)$.

$\left(\mathrm{H}_{1}\right) \mathrm{p}(\mathrm{x})>0, \int_{0}^{\infty} \frac{\mathrm{dx}}{\mathrm{p}(\mathrm{x})}=\infty, \mathrm{r}(\mathrm{x})>0, \mathrm{q}(\mathrm{x}) \geq 0$.

$\left(\mathrm{H}_{2}\right)$ For each solution $y$ of $(1), Q\left(y^{\prime \prime}, y\right)=p^{\prime \prime 2}+q y^{\prime \prime} y+r y^{2} \geq 0$.

The adjoint equation of (1.1) is

$$
\left(p z^{\prime \prime}+q z\right) "+r z=0 .
$$

Note that if $\mathrm{q}$ is a constant, then (1.1) is selfadjoint. A nontrivial solution 
of $(1.1)\{(1.2)\}$ is termed oscillatory if it changes signs for arbitrarily large values of $x$. We also say equation $(1.1)\{(1.2)\}$ is oscillatory whenever it has an oscillatory solution. Nonoscillatory solutions of (1.1) $\{(1.2)\}$ are those which fail to oscillate.

If it happens that all solutions of (1.1) $\{(1.2)\}$ are oscillatory we say that (1.1) $\{(1.2)\}$ is strongly oscillatory. If no solution oscillates then (1.1) $\{(1.2)\}$ is termed nonoscillatory. In either of these cases the solutions of (1.1) $\{(1.2)\}$ are said to have the same oscillatory character. Finally, we say that (1.1) $\{(1.2)\}$ is $(2,2)$-disconjugate if no nontrivial solution satisfies $y(a)=y^{\prime}(a)=0=y(b)=$ $y^{\prime}(b)$ for some $a$ and $b$ on $[0, \infty)$.

Some special cases of (1.1) have been studied in detail. In particular, the selfadjoint equation

$$
y^{i v}+r(x) y=0,
$$

has been studied extensively by Svec [1,2]. Svec examined the asymptotic properties of certain solutions and proved that all the solutions of (1.3) have the same oscillatory character. Leighton and Nehari [3] in their fundamental work concerning the more general selfadjoint equation

$$
\left(p(x) y^{\prime \prime}\right) "+r(x) y=0,
$$

obtained oscillation and nonoscillation criteria for solutions of (1.4). Schneider [4] proved that solutions of a $(2,2)$-disconjugate selfadjoint fourth order equation must all have the same oscillatory character. That the condition of selfadjointness is necessary can be seen by studying the following example:

EXAMPLE. The $(2,2)$-disconjugate nonselfadjoint Euler differential equation

$$
y^{i v}+\frac{1}{x^{2}} y^{\prime \prime}+\frac{1}{x^{4}} y=0
$$

has both oscillatory and nonoscillatory solutions, since the characteristic equation for (1.5), $r^{4}-6 r^{3}+12 r^{2}-7 r+1=0$ has exactly two real roots.

We also refer to some recent related work of Kreith [5].

\section{PRELIMINARY RESULTS.}

Consider the following functional defined on the solution space of (1.1):

$$
F[y(x)]=y(x)\left(p y^{\prime \prime}\right)^{\prime}(x)-y^{\prime}(x)\left(p y^{\prime \prime}\right)(x) \text {. }
$$


Computing the derivative of $F[y(x)]$ and making appropriate substitutions we find that

$$
F^{\prime}[y(x)]=-Q\left(y^{\prime \prime}(x), y(x)\right)
$$

from which it follows that $F[y(x)]$ is nonincreasing. This fact will be of considerable importance for the remainder of this work. The monotone property of $F[y(x)]$ implies that $(1.1)$ is $(2,2)$-disconjugate.

A solution $y(x)$ of $(1.1)$ is called a type I solution if $F[y(x)] \geq 0$ for all $x \geq 0$. A type II solution is one which fails to be type $I$. It is clear that (1.1) always has type II solutions. However, it is not obvious that nontrivial type I solutions exist. Before proceeding further, we establish the existence of such a solution.

THEOREM 2.1. There exists a nontrivial Type I solution.

PROOF. Suppose a $>0$. Let $\left\{x_{n}\right\}_{n=1}^{\infty}$ be an increasing sequence of numbers such that $a<x_{1}<x_{2} \ldots<x_{n} \ldots$ and $\lim _{n \rightarrow \infty} x_{n}=\infty$.

Let $y_{1}(x), y_{2}(x), y_{3}(x)$ be three linearly independent solutions of (1.1) which vanish at $\mathrm{x}=\mathrm{a}$, then 1 et

$$
u_{n}(x)=c_{1 n} y_{1}(x)+c_{2 n} y_{2}(x)+c_{3 n} y_{3}(x)
$$

be a solution of (1.1) satisfying

$$
\begin{aligned}
& u_{n}(a)=0 \\
& u_{n}\left(x_{n}\right)=u_{n}^{\prime}\left(x_{n}\right)=0 \\
& c_{1 n}^{2}+c_{2 n}^{2}+c_{3 n}^{2}=1
\end{aligned}
$$

Note that $F\left[u_{n}(x)\right]>0$ on $\left[0, x_{n}\right)$ for each $n$.

The sequences $\left\{c_{i n}\right\}_{n=1}^{\infty}$ for $i=1,2,3$ are bounded and hence there exists an increasing sequence of positive integers $\left\{n_{j}\right\}_{j=1}^{\infty}$ such that $\left\{c_{i n j}\right\}_{j=1}^{\infty}$ is convergent for $1=1,2,3$.

Let $c_{i}=\lim _{j \rightarrow \infty} c_{i n j}$, for $i=1,2,3$ and let $u(x)=c_{1} y_{1}(x)+c_{2} y_{2}(x)+c_{3} y_{3}(x)$. Since $c_{1}^{2}+c_{2}^{2}+c_{3}^{2}=1, u(x)$ is a nontrivial solution of (1.1).

We now show that $\mathrm{u}(\mathrm{x})$ is a type I solution. Suppose the contrary, then there is a number $c>$ a such that $F[u(c)]<0$. Since $u_{n j}(c) \rightarrow u(c)$, we can infer that 
$\left.\mathrm{F}\left[\mathrm{u}_{\mathrm{nj}}(\mathrm{c})\right] \rightarrow \mathrm{Flu}(\mathrm{c})\right]<0$. Choose a positive integer $\mathrm{N}$ such that for all $\mathrm{j}>\mathrm{N}$, $F\left[u_{n j}(c)\right]<0$ and $x_{n j}>c$. Since $F\left[u_{n j}(x)\right]$ is decreasing and $F\left[u_{n j}\left(x_{n j}\right)\right]=0$, for $j>N$, we get $0=F\left[u_{n j}\left(x_{n j}\right)\right]<F\left[u_{n j}(c)\right]<0$, a contradiction. Thus $u(x)$ is a type I solution of (1.1).

If $x=b$ is a number such that $u(b) \neq 0$, then using the same procedure we can construct a type I solution which is independent of $u(x)$. Consequently, (1.1) has at least two linearly independent type I solutions.

Before we proceed with some results on type II solutions we state the so-called "fundamental" lemma used so extensively in [5].

LEMMA. Suppose $\mathrm{u}, \mathrm{v} \in \mathrm{C}^{\prime}[\mathrm{a}, \mathrm{b}]$ with $\mathrm{u}\left(\mathrm{x}_{1}\right)=\mathrm{u}\left(\mathrm{x}_{2}\right)=0, \mathrm{a}<\mathrm{x}_{1}<\mathrm{x}_{1}<\mathrm{b}$, and $u(x) \neq 0$ for $x_{1}<x<x_{2}$. If $v(x) \neq 0$ on $\left[x_{1}, x_{2}\right]$ then some linear combination of $\mathrm{u}(\mathrm{x})$ and $\mathrm{v}(\mathrm{x})$ has a double zero in $\left(\mathrm{x}_{1}, \mathrm{x}_{2}\right)$.

THEOREM 2.2. A11 type II solutions have the same oscillatory character.

PROOF. Let $y_{a}(x)$ be the solution of (1.1) satisfying

$$
y_{a}(a)=y_{a}^{\prime}(a)=y_{a}^{\prime \prime}(a)=0,\left(p y_{a}^{\prime \prime}\right) \cdot(a)=1
$$

where $a>0$. We assert that if $y_{a}(x)$ is oscillatory and $b>a$, then $y_{b}(x)$ is oscillatory. Suppose the contrary, i.e. assume $\mathrm{y}_{a}(x)$ is oscillatory and that there is $a c>b$ such that $y_{b}(x)>0$ for $x \geq c$. We can assume without loss of generality that $\mathrm{y}_{\mathrm{a}}(\mathrm{b}) \geq 0$.

If $\alpha<\beta$ are consecutive zeros of $y_{a}$ in $(c, \infty)$ for which $y_{a}(x)>0$ for $\alpha<x<\beta$, then there is a constant $k>0$ such that the solution $z(x)=y_{a}(x)-k y_{b}(x)$ has a double zero in $(\alpha, \beta)$. Thus $F[z(b)]>0$, since $b<\alpha$. But $F[z(b)]=F\left[y_{a}(b)\right]-$ $\mathrm{ky}_{\mathrm{a}}(\mathrm{b})<0$. This contradiction proves our assertion.

Now let $u(x)$ be an arbitrary type II solution of (1.1). If $u(x)$ is nonoscillatory and positive on some interval $[e, \infty)$ where $e$ is chosen large enough so that $F[u(e)]<0$. The $y_{e}(x)$ is oscillatory, assuming $y_{a}(x)$ oscillatory. Consequently, there is a linear combination $\mathrm{W}(\mathrm{x})=\mathrm{y}_{\mathrm{e}}(\mathrm{x})-\mathrm{ku}(\mathrm{x}), \mathrm{k}>0$, have a double zero at $e_{1} \geq e$. Thus $F\left[W\left(e_{1}\right)\right]=0$. But $F[W(e)]=-k U(e)+k^{2} F[U(e)]<0$, a contradiction since $e_{1} \geq e$ and $F[W(x)]$ is decreasing.

Thus, an arbitrary type II solution is oscillatory whenever $y_{a}(x)$ is oscillatory 
for some $a \geq 0$. In a similar manner it can be shown that the existence of an oscillatory type II solution implies that $y_{a}(x)$ is oscillatory and our theorem follows.

\section{PROPERTIES OF NONOSCILLATORY SOLUTIONS.}

We now focus on the asymptotic behavior of nonoscillatory solutions. We begin by relating the zero behavior of a solution $y(x)$ with the zero behavior of $y^{\prime \prime}(x)$. This theorem is essential to the study of the nonoscillatory solutions of (1.1). Note the role played by the functional $F[y(x)]$.

THEOREM 3.1. Let $y(x)$ be a nontrivial solution of (1.1). There exists a point $c>0$ such that either $F[y(x)] \neq 0$ on $[c, \infty)$ or $F[y(x)] \equiv 0$ on $[c, \infty)$. If $\mathrm{F}[\mathrm{y}(\mathrm{x})] \neq 0$ on $[c, \infty)$, then the zeros of $\mathrm{y}(\mathrm{x})$ and $\mathrm{y}^{\prime \prime}(\mathrm{x})$ separate on $[c, \infty)$. If $\mathrm{F}[\mathrm{y}(\mathrm{x})] \equiv 0$ on $[c, \infty)$, then the zeros of $\mathrm{y}(\mathrm{x})$ and $\mathrm{y}^{\prime \prime}(\mathrm{x})$ coincide.

PROOF. Suppose $\mathrm{F}[\mathrm{y}(\mathrm{x})] \neq 0$ for $\mathrm{all} \mathrm{x}>\mathrm{c}$ and let $\mathrm{x}_{0}<\mathrm{x}_{1}$ be consecutive zeros of $y(x)$ on $[c, \infty)$. Assume $y^{\prime \prime}(x)>0$ on $\left[x_{0}, x_{1}\right]$. Then

$$
\left(\frac{y}{p^{\prime \prime}}\right)^{\prime}=-\frac{F[y]}{\left(p y^{\prime \prime}\right)^{2}}
$$

Integrating (3.1) from $x_{0}$ to $x_{1}$ yields

$$
0=\frac{y\left(x_{1}\right)}{\left(p y^{\prime \prime}\right)\left(x_{1}\right)}-\frac{y\left(x_{o}\right)}{\left(p y^{\prime \prime}\right)\left(x_{0}\right)}=-\int_{x_{0}}^{x_{1}} \frac{F[y(t)] d t}{\left(p y^{\prime \prime}\right)^{2}(t)} \neq 0
$$

which is a contradiction. Thus between consecutive zeros of $y(x), y^{\prime \prime}(x)$ has a zero. Exchanging the roles of $y(x)$ and $y^{\prime \prime}(x)$ we can prove that the zeros of $y(x)$ separate the zeros of $y^{\prime \prime}(x)$ for sufficiently large $x$.

Now consider the case $F[y(x)] \equiv 0$ on $[c, \infty)$. Then

$$
F^{\prime}[y(x)]=-p(x) y^{\prime \prime 2}(x)-q(x) y^{\prime \prime}(x) y(x)-r(x) y^{2}(x) \equiv 0
$$

on $(c, \infty)$. From which we see that if $d>c$ and $y(d)=0$, then $y^{\prime \prime}(d)=0$. Similarly if $y^{\prime \prime}(d)=0$, then $y(d)=0$. So the zeros of $y(x)$ and $y^{\prime \prime}(x)$ coincide on $(c, \infty)$. This completes the proof of our theorem.

For nonoscillatory solutions of (1.1) it follows immediately that there is a number $c$ such that 


$$
y(x) y^{\prime}(x) y^{\prime \prime}(x) \neq 0 \text { for all } x \geq c .
$$

More explicit information concerning the nonoscillatory solutions of (1.1) can now be given.

THEOREM 3.2. Let $y(x)$ be a nonoscillatory solution of (1.1). Then there exists a number $a>0$ such that for all $\mathrm{x}>\mathrm{a}$, either

$$
\text { (i) } \operatorname{sgn} y(x)=\operatorname{sgn} y^{\prime}(x)=\operatorname{sgn} y^{\prime \prime}(x)=\operatorname{sgn}\left(p y^{\prime \prime}\right)^{\prime}(x)
$$

or

$$
\text { (ii) } \operatorname{sgn} y(x)=\operatorname{sgn} y^{\prime}(x) \neq \operatorname{sgn} y^{\prime \prime}(x) \text {. }
$$

Moreover, if $y(x)$ satisfies (i) then $\lim _{x \rightarrow \infty}|y(x)|=\lim _{x \rightarrow \infty}\left|y^{\prime}(x)\right|=\infty$.

In case $y(x)$ satisfies (ii) then $\lim _{x \rightarrow \infty} y^{\prime}(x)$ exists and $\lim _{x \rightarrow \infty} \inf \left|y^{\prime \prime}(x)\right|=0$.

THEOREM 3.3. A nonoscillatory solution $y(x)$ of $(1.1)$ is type $I$ if and only if

$$
\operatorname{sgn} y(x)=\operatorname{sgn} y^{\prime}(x) \neq \operatorname{sgn} y^{\prime \prime}(x),
$$

for all x sufficiently large.

PROOF. Let $y(x)$ be a nonoscillatory solution satisfying

$$
\mathrm{y}(\mathrm{x})>0, \mathrm{y}^{\prime}(\mathrm{x})>0, \mathrm{y}^{\prime \prime}(\mathrm{x})<0 .
$$

Consider the functional $F[y(x)]=y(x)\left(p y^{\prime \prime}\right)^{\prime}(x)-y^{\prime}(x)\left(p y^{\prime \prime}\right)(x)$.

If there is a number $x>c$ such that $F[y(c)]<0$, then $F[y(x)]<0$ on $[c, \infty)$, but

$$
\mathrm{y}(\mathrm{x})>0, \mathrm{y}^{\prime}(\mathrm{x})>0 \text { and } \mathrm{y}^{\prime \prime}(\mathrm{x})<0
$$

for $\mathrm{x}>\mathrm{c}$, together with $\mathrm{F}[\mathrm{y}(\mathrm{x})]<0$ for $\mathrm{x}>\mathrm{c}$, implies $\left.(\mathrm{py})^{\prime \prime}\right)^{\prime}(\mathrm{x})<0$ for $\mathrm{x}>\mathrm{c}$. This inequality, however, will force $y(x)$ to become negative eventually, contradicting $y(x)>0$ on $[c, \infty)$. Thus $F[y(x)] \geq 0$ for all $x$. To prove the converse, let $y(x)$ be a nonoscillatory type I solution, then from a preceding theorem there is a number b such that either

$$
\begin{aligned}
& \text { (i) } \operatorname{sgn} y(x)=\operatorname{sgn} y^{\prime}(x)=\operatorname{sgn} y^{\prime \prime}(x)=\operatorname{sgn}\left(p y^{\prime \prime}\right){ }^{\prime}(x) \text {, or } \\
& \text { (ii) } \operatorname{sgn} y(x)=\operatorname{sgn} y^{\prime}(x) \neq \operatorname{sgn} y^{\prime \prime}(x) \text { for all } x \geq b \text {. }
\end{aligned}
$$

We will show that being type I excludes the possibility of (1.1). Suppose the contrary.

Let $y(x)$ be a solution of (1.1) satisfying (i) and suppose $F[y(x)]>0$ on $[c, \infty)$. 
Then $F[y(x)]>0$ and $y(x)>0$ implies

$$
(\text { py") })^{\prime}(\mathrm{x})>\mathrm{y}^{\prime}(\mathrm{x}) \cdot \frac{(\mathrm{py})(\mathrm{x})}{\mathrm{y}(\mathrm{x})}
$$

The positivity of $\mathrm{F}[\mathrm{y}(\mathrm{x})]$ also implies $\frac{(\mathrm{py})(\mathrm{x})}{\mathrm{y}(\mathrm{x})}$ is positive and increasing, so (3.2) implies (py")' $(x) \rightarrow \infty$ as $x \rightarrow \infty$. But this is impossible since (py")"( $x)<0$ on $[c, \infty)$. Consequently (i) is inconsistent with being type $I$.

COROLLARY. A nonoscillatory solution $y(x)$ is type II if and only if there exists $c>0$ such that

$$
\operatorname{sgn} y(x)=\operatorname{sgn} y^{\prime}(x)=\operatorname{sgn} y^{\prime \prime}(x)=\operatorname{sgn}\left(p y^{\prime \prime}\right)^{\prime}(x)
$$

for all $x>c$.

We now compare $y(x)$ and $(p y ")(x)$ for nonoscillatory solutions. The proofs are easy and will be omitted.

THEOREM 3.4. Let $y(x)$ be a nonoscillatory solution of $(1.1)$. Then $\lim _{x \rightarrow \infty} \frac{(p y ")(x)}{y(x)}$ exists. Moreover, in case $y(x)$ is type $I$, then $\lim _{x \rightarrow \infty} \frac{(p y ")(x)}{y(x)}=0$.

The next result yields a necessary and sufficient condition for (1.1) to be nonoscillatory.

THEOREM 3.5. These are equivalent:

(A) Equation (1.1) is nonoscillatory.

(B) Equation (1.1) has both a type I nonoscillatory solution and a type II nonoscillatory solution.

PROOF. We prove only (B) implies (A) since (A) implies (B) is obvious. Suppose (1.1) has an oscillatory solution $u(x)$. Then it follows from Theorem 2.2 the $u(x)$ is type I. Let $z(x)$ be a type I nonoscillatory solution. Then there is a combination $y(x)=k z(x)+u(x), k \neq 0$, which has a double zero. Note that $y(x)$ is nonoscillatory, since all type II solutions are nonoscillatory. Solving for $u(x)$ we find that $u(x)=y(x)-k z(x)$. However, $\underset{x \rightarrow \infty}{\operatorname{iim}}\left|y^{\prime}(x)\right| \rightarrow \infty$ and $z^{\prime}(x)$ is bounded on some ray $[c, \infty)$, so $u(x)$ is not oscillatory. This contradiction shows that (1.1) is nonoscillatory.

EXAMPLE. The equation

$$
y^{i v}+\frac{5}{48 x^{2}} y^{\prime \prime}+\frac{35}{64 x^{4}} y=0
$$


has both a type I nonoscillatory solution $y(x)=x^{3 / 4}$ and a type II solution nonoscillatory solution $y(x)=x^{5 / 2}$, hence by Theorem 3.5, Equation (3.3) is nonoscillatory.

We now examine some relationship between (1.1) and 1ts adjoint (1.2) in the form

$$
\left(p z^{\prime \prime}+q z\right) "+r z=0 .
$$

As a notational convenience we define the differential operators

$$
\mathrm{D}_{2} \mathrm{z}=\mathrm{pz} "+\mathrm{qz}, \mathrm{D}_{3} \mathrm{z}=\left(\mathrm{D}_{2} \mathrm{z}\right)^{\prime}, \mathrm{D}_{4} \mathrm{z}=\left(\mathrm{D}_{3} \mathrm{z}\right)^{\prime} .
$$

For a solution of $(1.2)$, assuming $Q\left(z^{\prime \prime}, z\right)=p z^{\prime 2}+q z^{\prime \prime} z+r z^{2} \geq 0$ we see that the functional

$$
G[z(x)]=z(x) D_{3} z(x)-z^{\prime}(x) D_{2} z(x)
$$

is nonincreasing. This follows immediately from the fact that $G^{\prime}[z]=-Q\left(z^{\prime \prime}, z\right) \leq 0$.

Proceeding as before, we shall say that a solution $z(x)$ of $(1.2)$ is a type I* or If and only if $G[z(x)] \geq 0$ for all $x \geq 0$. All other solutions of (1.2) will be called type II* solutions.

THEOREM 3.6. All type II* solutions have the same oscillatory character. The proof of this theorem is analogous to the proof of Theorem 2.2 and will be omitted.

The following lemma will be used to establish a connection between the oscillation of (1.1) and (1.2).

LEMMA. Suppose $\mathrm{y}$ is a solution of $(1.1)$ and $z$ is a solution of (1.2), then

$$
z\left(p(x) y^{\prime \prime}\right)^{\prime}-z^{\prime}\left(p(x) y^{\prime \prime}\right)+y^{\prime} D_{2} z-y_{3} z \equiv k_{1}
$$

$\mathrm{k}_{1}$, constant.

THEOREM 3.7. If type II* solutions oscillate, then type I solutions osclllate.

PROOF. Suppose type II* solutions are oscillatory while $y(x)$ is a type I nonoscillatory solution of (1.1). Assume $y(x)>0$ on $[a, \infty)$ for some $a \geq 0$. Let $z_{a}(x)$ denote the solution of (1.2) satisfying

$$
z_{a}(a)=z_{a}^{\prime}(a)=D_{2} z_{a}(a)=0, D_{3} z_{a}(a)=1 .
$$

Then $z_{a}(x)$ is oscillatory. Let $\alpha$ and $\beta$ be consecutive zeros of $z_{a}(x)$ and suppose $z_{a}(x)>0$ on $(\alpha, \beta)$. Consider the following

$$
\left(\frac{D_{2} z_{a}}{y}\right)^{\prime}=\frac{y D_{3} z_{a}-y^{\prime} D_{2} z_{a}}{y^{2}} .
$$


From the previous lemma

$$
\mathrm{yD}_{3} z_{a}-\mathrm{y}^{\prime} \mathrm{D}_{2} \mathrm{z}_{\mathrm{a}}+\mathrm{z}_{\mathrm{a}}^{\prime}\left(\mathrm{py} \mathrm{y}^{\prime \prime}\right)-\mathrm{z}_{\mathrm{a}}\left(\mathrm{py} \mathrm{y}^{\prime \prime}\right)^{\prime} \equiv \mathrm{k} \text {. }
$$

Thus we see that $\mathrm{yD}_{3} z_{a}-y^{\prime} \mathrm{D}_{2} z_{a}=z_{a}\left(p y^{\prime \prime}\right)^{\prime}-z_{a}^{\prime}\left(p y^{\prime \prime}\right)+k$ so making a substitution in (3.4) we obtain

$$
\left(\frac{D_{2} z_{a}}{y}\right)^{\prime}=\frac{z_{a}\left(p y^{\prime \prime}\right)^{\prime}-z_{a}^{\prime}\left(p y^{\prime \prime}\right)+k}{y^{2}},
$$

Integrating (3.4a) by parts from $x=\alpha$ to $x=\beta$ we get

$$
\frac{\left(p z_{a}^{\prime \prime}\right)(\beta)}{y(\beta)}-\frac{\left(p z_{a}^{\prime \prime}\right)(\alpha)}{y(\alpha)}=\int_{\beta}^{\alpha} \frac{2 z_{a}^{(x)} F[y(x)]+k y(x)}{y^{3}(x)} d x,
$$

where $k=y(a) \geq 0$.

Since $z_{a}(x)>0$ on $(\alpha, \beta), G\left[z_{a}(\alpha)\right]=-p(\alpha)\left(z_{a}^{\prime} z_{a}^{\prime \prime}\right)(\alpha)<0$ and $G\left[z_{a}(\beta)\right]=$ $-p(\beta) z_{a}^{\prime}(\beta) z_{a}^{\prime \prime}(\beta)<0$, we conclude that $z_{a}^{\prime}(\alpha)>0, z_{a}^{\prime \prime}(\alpha)>0, z_{a}^{\prime}(\beta)<0$ and $z_{a}^{\prime \prime}(\beta)<0$. This implies that the left side of (3.5) is negative, but note that the right side of (3.5) is positive. This contradiction proves that $y(x)$ must oscillate.

COROLLARY. All type I solutions have the same oscillatory character. The companion Theorem of Theorem 3.7 is also true. We state it without proof.

THEOREM 3.8. If type I* solutions oscillate, then type II solutions oscillate. Immediately it follows that if all solutions of (1.1) have the same oscillatory character then all solutions of (1.2) have the same oscillatory character.

OSCILIATION CRITERIA. In this section we establish some sufficient conditions for all solutions of (1.1) to be oscillatory. One such condition makes use of the nonoscillation numbers $r_{1 j}$ studied by Peterson [6]. We describe these briefly. Let $i$ and $j$ be nonnegative integers such that $i+j=4$. A solution $y(x)$ is said to have an $(1, j)$-distribution of zeros on an interval I if there are two numbers $x_{1}$ and $x_{j}$ in $I$ such that $x_{1}<x_{j}$ and $y(x)$ has a zero of multiplicity at least 1 at $x_{i}$ and a zero of multiplicity at least $j$ at $x_{j}$ and $y(x) \neq 0$ in $\left(x_{i}, x_{j}\right)$. If on $[t, \infty)$ there are solutions with $(i, j)$-distributions of zero then $r_{1 j}(t)$ for equation (1.1) is the infimum of the numbers $b>t$ such that there exists a solution $y(x)$ 
of (1.1) having an $(i, j)$ distribution of zeros on $[t, b)$. If no such distribution exists on $I$ then we write $r_{i j}=\infty$ on $I$.

THEOREM 3.9. If $r_{31}(t)<\infty$ and $r_{13}(t)<\infty$ for all $t$ on some ray $[\alpha, \infty), \alpha<0$, then all solutions of $(1.1)$ are oscillatory.

PROOF. We only show type I solutions oscillate, since the proof is similar to show type II solutions oscillate.

Suppose $y(x)>0$ on $[b, \infty)$ for some $b \geq \alpha$ and $F[y(x)]>0$ on $[b, \infty)$. Let $u(x)$ be a nontrivial solution of (1.1) having a $(1,3)$-distribution at $x=c$ and $x=d$, $b<c<d$; we assume $\left(p u^{\prime \prime}\right)^{\prime}(d)=1$. Since $y(x)>0$ on $[b, \infty)$ and $u(x)<0$ on $(c, d)$, there is a positive constant $k$ such that $v(x)=y(x)+k u(x)$ has a double zero in $(c, d)$. Since $F[v(x)]$ is decreasing and $F[v(x)]$ vanishes in $(c, d)$ we conclude that $\mathrm{F}[\mathrm{v}(\mathrm{d})]<0$. But $\mathrm{F}[\mathrm{v}(\mathrm{d})]=\mathrm{F}[\mathrm{y}(\mathrm{d})]+\mathrm{k} \mathrm{y}(\mathrm{d})>0$. This contradiction proves that $y(x)$ must have a zero between $x=c$ and $x=d$. Now, since $r_{13}(t)$ exists for every $t>0$ it is easy to show that type I solutions have infinitely many zeros on $[\alpha, \infty)$. THEOREM 3.10. If $\int_{0}^{\infty} r(x) d x=\infty$ and $\frac{q(x)}{p(x)} \leq r(x)$ and $p(x)$ bounded, then all solutions of (1.2), hence (1.1), are oscillatory.

PROOF. Suppose $z(x)$ is a nonoscillatory solution of (1.2) satisfying $z(x)>0$ on $[a, \infty)$. Then $D_{4} z(x)<0$ on $[a, \infty)$ and $D_{3} z(x)$ is decreasing. If $D_{3} z(b)<0$ for some $b>a$ then $D_{3} z(x)<0$ for $x>b$ and $D_{2} z(x) \rightarrow-\infty$. Choose $c \geq b$ such that $p(x) z^{\prime \prime}(x) \leq-1$ for $x>c$. Then $z^{\prime \prime}(x) \leq-\frac{1}{p(x)}$ on $[c, \infty)$. Integrating this inequality we obtain $z^{\prime}(x) \leq z^{\prime}(c)-\int_{c}^{\bar{x}} \frac{d t}{p(t)}$, which implies $z^{\prime}(x) \rightarrow-\infty$ as $x \rightarrow \infty$. But this contradicts $z(x)>0$ on $[c, \infty)$. So $D_{3} z(x)>0$ for all $x>a$. Note however that $\mathrm{D}_{3} z(\mathrm{x})>0$ and decreasing implies

$$
\int_{a}^{\infty} r(x) z(x) d x<\infty .
$$

Now let us examine $\mathrm{D}_{2} \mathrm{z}(\mathrm{x})$. We know $\mathrm{D}_{2} \mathrm{z}(\mathrm{x})$ is increasing. If $\mathrm{D}_{2} \mathrm{z}(\mathrm{x})<0$ on $[\mathrm{b}, \infty)$, $b \geq a$, then $z(x)$ is increasing on $[c, \infty)$ for some $c>b$.

So $z(x)>z(c)$ for $x>c$ and

$$
D_{3} z(x)-D_{3} z(c)=-\int_{c}^{x} r(t) z(t) d t<-z(c) \int_{c}^{x} r(t) d t
$$


As $x \rightarrow \infty, \int_{C}^{x} r(t) d t \rightarrow \infty$, but this forces $D_{3} z(x)$ to be negative at some point greater than a, clearly a contradiction. Therefore, $D_{2} z(x)<0$ on some ray $[b, \infty)$ is impossible. Now consider the case where $D_{2} z(x)>0$ on $[b, \infty)$. If $M$ is an upper bound for $\mathrm{p}(\mathrm{x})$ then using our hypothesis we obtain

$$
\begin{aligned}
& M\left[z^{\prime \prime}+r(x) z\right] \geq M\left(z^{\prime \prime}(x)+\frac{q(x)}{p(x)} z(x)\right) \geq p(x)\left[z^{\prime \prime}(x)+\frac{q(x)}{p(x)} z(x)\right]= \\
& D_{2} z(x)>D_{2} z(b) \text { for } x>b .
\end{aligned}
$$

Integrating (3.6) we see that

$$
0<D_{2} z(b)(x-b)<M \int_{b}^{x} r(t) z(t) d t+M z^{\prime}(x)-M z^{\prime}(b)
$$

which implies $z^{\prime}(x) \rightarrow \infty$ as $x \rightarrow \infty$. Thus $z(x)$ is increasing on some ray $[d, \infty)$, $\mathrm{d} \geq \mathrm{c}$

But this leads us to a contradiction, since $\int_{a}^{\infty} r(x) d x=\infty$. Consequent1y, a11 solutions of $(1.2)$ are oscillatory.

THEOREM 3.11. If $\int_{0}^{\infty} \mathrm{x} r(\mathrm{x}) \mathrm{dx}=\infty$ and $\mathrm{xq}(\mathrm{x}) \leq \mathrm{M}$, then a11 solutions (1.1) are oscillatory.

PROOF. Suppose (1.1) has a nonoscillatory solution $y(x)$. Assume without loss of generality that $y(x)>0$, for $a 11 x>b$. If $y(x)$ is a type I solution then there is a number $c$ such that for all $x>c$

$$
y(x)>0, y^{\prime}(x)>0, y^{\prime \prime}(x)<0 \text {. }
$$

Multiplying (1.1) by $\mathrm{x}$ and integrating we get

$$
x\left(p y^{\prime \prime}\right)^{\prime}(x)-c\left(p y^{\prime \prime}\right)^{\prime}(c)+\int_{c}^{x} t q(t) y^{\prime \prime}(t) d t+\int_{c}^{x} t r(t) y(t) d t=0 .
$$

Let $M$ be an upper bound for $x q(x)$. Then we have

$$
x\left(p y^{\prime \prime}\right) '(x)-c\left(p u^{\prime \prime}\right)^{\prime}(c)+M\left[y^{\prime}(x)-\dot{y}^{\prime}(c)\right]+\int_{c}^{x} t r(t) y(t) d t \leq 0 .
$$

Since $\mathrm{y}(\mathrm{x})$ is positive and increasing the integral tends to infinity. Moreover, since $y^{\prime \prime}(x)<0, y^{\prime}(x)$ has a finite limit. Thus (py")(x) is negative for large $x$ and (py") $(x)$ is decreasing. Let $B<0$ be a number such that $y^{\prime \prime}(x)<\frac{B}{p(x)}$ for $x>c$. Integrating this inequality from $c$ to $x$ we conclude that $\lim _{x^{\prime} \rightarrow \infty} y^{\prime}(x)=-\infty$, which is absurd since $\mathrm{y}^{\prime}(\mathrm{x})$ is bounded.

If $y(x)$ is a positive type II solution, then since $y^{\prime}(x)$ and $y^{\prime \prime}(x)$ are 
positive there exists a positive number in such that $y(x) \geq m x$ for large $x$. Substituting in (1.1) and integrating we get

$$
\left(p^{\prime \prime}\right)^{\prime}(x)-\left(p y^{\prime \prime}\right)^{\prime}(c) \leq-\int_{C}^{x} q(t) y^{\prime \prime}(t) d t-M \int_{C}^{x} t r(t) d t \text {. }
$$

We conclude from this inequality that $\left(\mathrm{py} \mathrm{y}^{\prime}\right)^{\prime}(\mathrm{x}) \rightarrow-\infty$ as $\mathrm{x} \rightarrow \infty$, a contradiction. Thus type II solutions must be oscillatory.

\section{REFERENCES}

1. SVEC, MARKO Sur Une Propriete des Integrales de $\ell^{\prime}$ Equation $\mathrm{y}^{(\mathrm{n})}+\mathrm{Q}(\mathrm{x}) \mathrm{y}=0, \mathrm{n}=3,4, \underline{\text { Czech. Math. J } 7(82)} 1957,450-462$.

2. SVEC, MARKO Sur le Comportment Asymptotic des Integrales de $l^{\prime}$ Equation Differentielle $\mathrm{y}^{(4)}+\mathrm{Q}(\mathrm{x}) \mathrm{y}=0$. Czech. Math. J. 8 (1958), 230-245.

3. LEIGHTON, W. and NEHARI, $z$. On the Oscillation of Solutions of Selfadjoint Linear Differential Equations of the Fourth Order, Trans. Amer. Math. Soc. $\underline{89}(1958), 325-377$.

4. SCHNEIDER, L. Oscillation Properties of the $(2,2)$-Disconjugate Fourth Order Selfadjoint Differential Equation, Proc. Amer. Math. Soc. 28 (1971), 545-550.

5. KREITH, K. Rotation Properties of Adjoint Pairs of Differential Systems, Proc. Royal Soc. Edinburgh 75A (1975), 149-155.

6. PETERSON, A. Distribution of Zeros of Fourth Order Differential Equation, Pacific J. Math. 30 (1969), 751-764.

7. TAYLOR, W.E., JR. and ETGEN, G.J. On the Oscillation of a Class of Fourth Order Differential Equations, Rocky Mt. J. Math. $\underline{6}$ (1976), 71-84. 


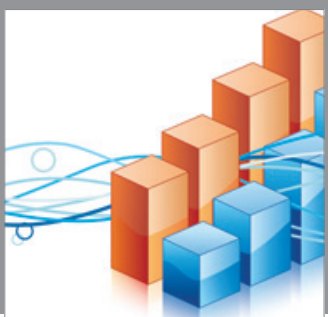

Advances in

Operations Research

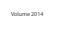

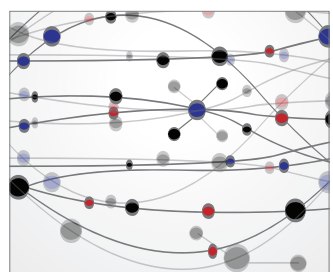

\section{The Scientific} World Journal
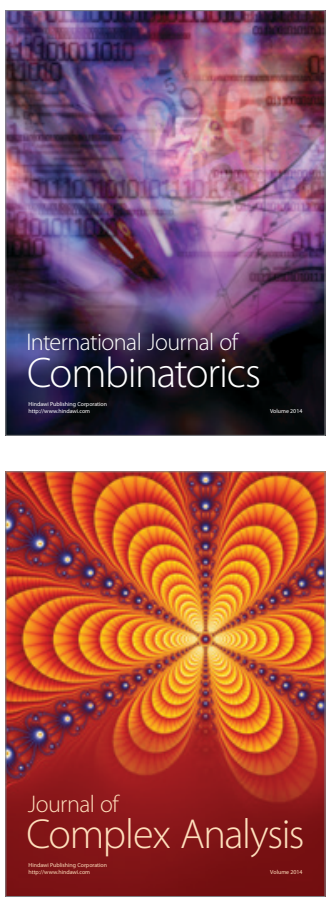

International Journal of

Mathematics and

Mathematical

Sciences
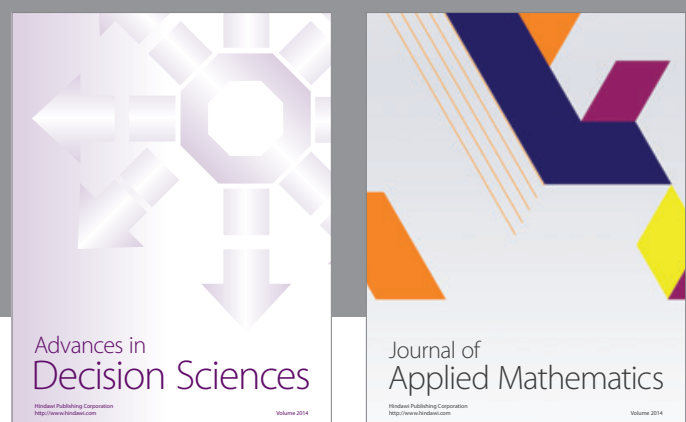

Journal of

Applied Mathematics
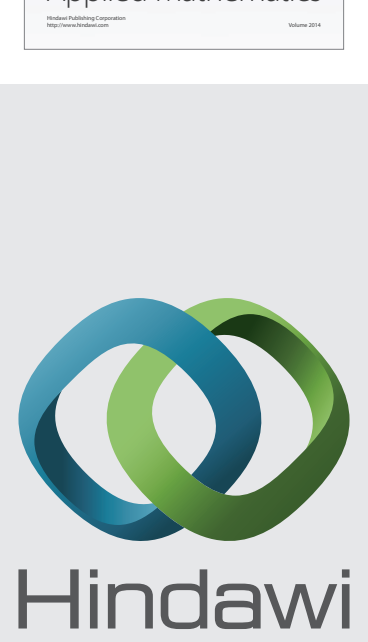

Submit your manuscripts at http://www.hindawi.com
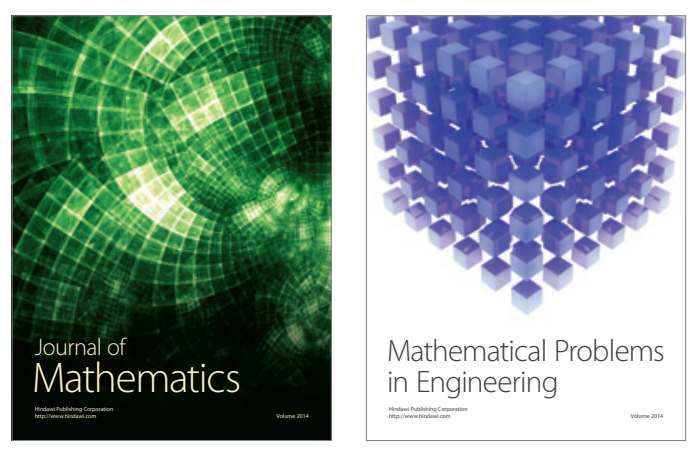

Mathematical Problems in Engineering
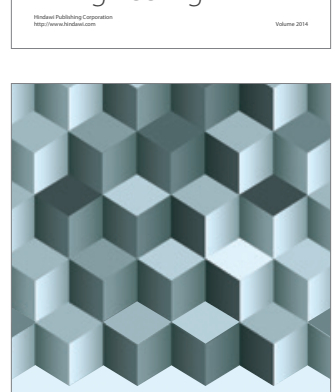

Journal of

Function Spaces
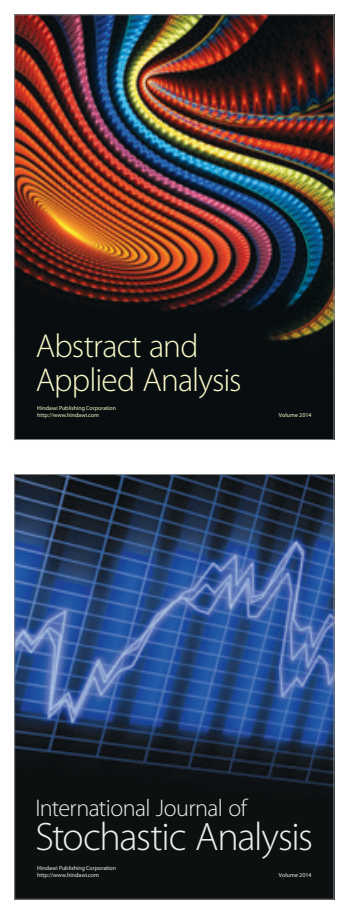

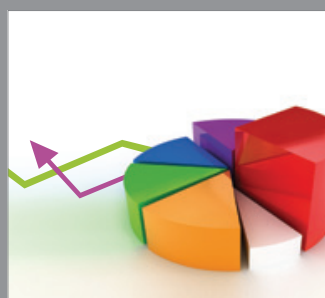

ournal of

Probability and Statistics

Promensencen
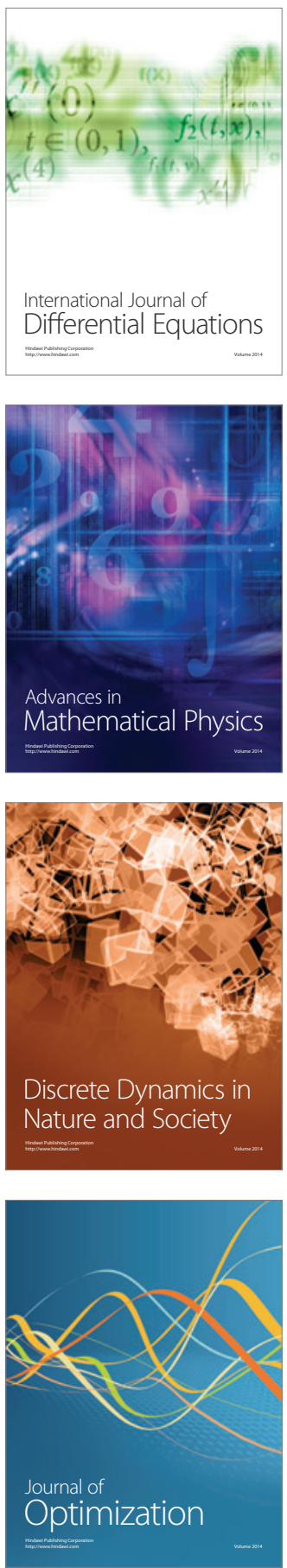